\title{
Interféromètre S.I.S.A.M. Présentation générale et applications
}

\author{
I. Verrier, G. Brun et J.-P. Goure \\ Laboratoire Traitement du Signal et Instrumentation, UMR 5516 du CNRS, 23 rue du \\ Docteur Paul Michelon, 42023 Saint-Etienne cedex 2, France
}

\begin{abstract}
Résumé : L'interféromètre S.I.S.A.M. (Spectromètre Interférentiel à Sélection par l'Amplitude de la Modulation) est un dispositif permettant la corrélation en amplitude de champs optiques. Cet interféromètre fonctionne en lumière large bande spectrale et donne accès à la mesure directe de différences de trajets optiques avec une grande dynamique. Les applications possibles sont par exemple la mesure de temps de propagation des modes dans les fibres optiques ou la mesure de faibles épaisseurs dans des milieux d'indice de réfraction variable.
\end{abstract}

\section{PRINCIPE DE FONCTIONNEMENT}

Le S.I.S.A.M., décrit par Pierre Connes [1, 2] dans les années 1960, est un interféromètre de Michelson dont les miroirs sont remplacés par des réseaux de diffraction fonctionnant dans l'ordre 1 (Figure 1). Un champ optique de référence en incidence $\theta$ est diffracté par l'un des réseaux. Les champs à étudier sont envoyés sur l'autre réseau sous la même incidence $\theta$. Ils sont retardés les uns par rapport aux autres et l'on veut déterminer leur séparation temporelle (ou les trajets optiques correspondants).

Pour une longueur d'onde, chaque réseau retarde les différents champs optiques qui parcourent, sur la section transverse du faisceau diffracté correspondant, des chemins optiques différents. Les différentes longueurs d'onde de la source lumineuse à large spectre, induisent grâce aux réseaux, des différences de marche spécifiques sur le profil transversal de chaque faisceau [3].

Une lame séparatrice et une lentille permettent d'effectuer en sortie du S.I.S.A.M., sur une barrette $C C D$, la corrélation des différents champs à tester avec celui de référence. La lentille est positionnée de manière à former sur la barrette, l'image du centre de chaque réseau (Figure $1)$.

\section{RÉSULTATS ET MESURES EFFECTUÉES}

Avec le dispositif S.I.S.A.M., nous sommes donc capables de mesurer des différences de temps de propagation $\Delta \tau$ ou des différences de trajets optiques $\Delta \delta$ sur les champs issus de la voie de mesure. Ces grandeurs à mesurer sont converties en variable spatiale $X_{0}^{\prime}$ sur le détecteur. En effet, à l'issue du S.I.S.A.M., I'intensité lumineuse sur le détecteur est donnée par une relation de la forme : 


$$
\xi(X)=A+B \operatorname{sinc}\left[\pi \Delta v f\left(X-X_{1}\right)\right]+C \operatorname{sinc}\left[\pi \Delta v f\left(X-X_{2}\right)\right]
$$

$A, B$ et $C$ désignent des constantes qui rendent compte notamment du spectre en fréquence de la source, $X$ représente la variable spatiale décrivant la répartition de l'intensité lumineuse sur le détecteur linéaire et $\Delta v$ est la plage de fréquences explorée par le laser.

La quantité $X_{0}^{\prime}$ est donnée par la relation $X_{0}^{\prime}=X_{2}-X_{1}$ et conduit à $X^{\prime}{ }_{0}=\frac{\gamma \Delta \delta}{c Q}$ si on mesure un trajet $\Delta \delta$ ou à $X_{0}^{*}=\frac{\gamma \Delta \tau}{Q}$ si on mesure une différence de temps $\Delta \tau$.

Le facteur $Q$ est relié aux paramètres opto-géométriques du montage :

$$
Q=\frac{2 N_{0}}{v_{0} \cos \theta_{0}^{\prime}}
$$

$\gamma$ représente le grandissement transversal de la lentille, $v_{0}$ est la fréquence centrale de la source lumineuse, $\theta^{\prime}$ désigne l'angle de diffraction après chaque réseau pour la fréquence $v_{0}$ et $N_{0}$ est le nombre de traits par millimètre de chaque réseau.

Une première application de cette méthode a permis de mesurer les différences de temps de propagation de modes successifs dans une fibre optique faiblement multimodale [4] (Figure 2). La différence de temps de propagation mesurée entre les deux premiers modes correspond à $\Delta \tau=700 \pm 50 \mathrm{fs}$.

Une deuxième application concerne la mesure d'épaisseur d'un système multicouche constitué par un empilement de lamelles de verre (Figure 3). La configuration du dispositif permet de simuler un "gradient d'épaisseur" qu'il est possible de reconstituer par la technique de corrélation de champs précédemment décrite.

La dynamique de mesure de ce dispositif est grande et sa résolution encore insuffisante peut être très nettement améliorée. L'étape suivante de notre travail consistera donc à accroître les performances du système en termes de résolution puis à le mettre en œuvre pour la détection d'objets transparents d'épaisseur ou d'indice variables placés dans des milieux fortement diffusants.

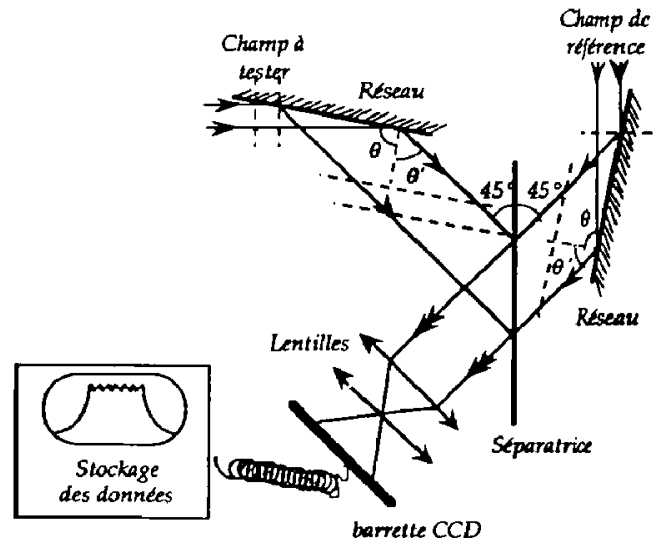

Figure 1 : Interféromètre S.I.S.A.M.

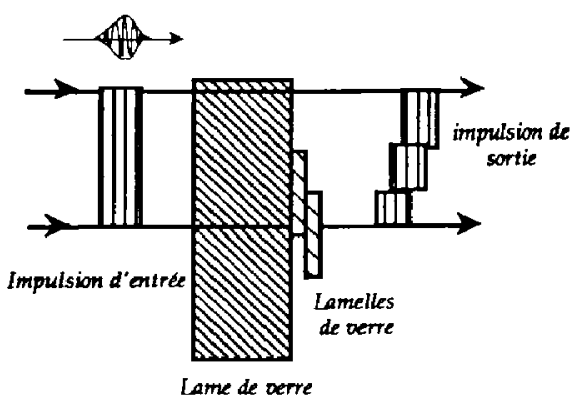

Figure 3 : Mesures d'épaisseur d'un système multicouche 


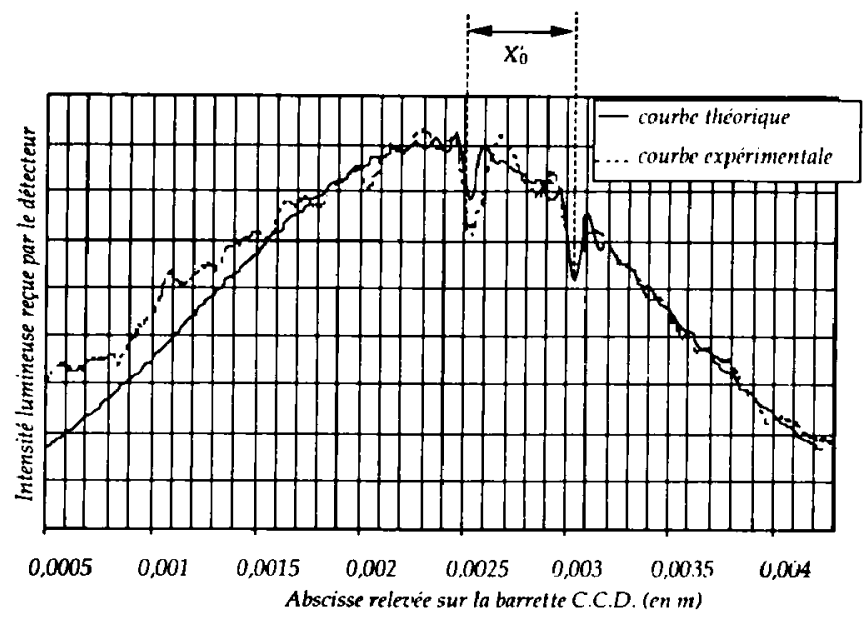

Figure 2 : Courbes théorique et expérimentale des mesures de délais de groupe dans des fibres optiques

\section{Remerciements.}

Les auteurs remercient Messieurs C. Froehly et P. Faugeras (Limoges) pour leur soutien et leurs suggestions concernant ce travail.

\section{Références}

[1] Connes P., Rev. d'Opt. Théor. et Appl., 38 (1959), 157-201.

[2] Connes P., Rev. d'Opt. Théor. et Appl., 39 (1960), 402-436.

[3] Brun G., Verrier I., Barthélémy A., Froehly C. et Goure J.P., J. of Opt. Com., 13 (1992), 134-139.

[4] Brun G., Verrier I., Ramos M., Goure J.P., Ottavi P. et Lambert A.M., Appl. Opt., 35 (1996), 1129-1134. 